\title{
Schizophrenia: brain morphology and treatment aspects
}

\author{
Andrea Schmitt $\cdot$ Peter Falkai
}

Published online: 10 January 2013

(c) Springer-Verlag Berlin Heidelberg 2013

Since the first findings of enlarged ventricles in computer tomography studies and with the development of MRIbased segmentation, brain morphology findings in schizophrenia point to abnormal structural findings in several brain regions involved in neuronal circuits [1]. One of the often cited environmental risk factors in schizophrenia is cannabis abuse. In an invited review, Malchow et al. [2], however, found inconclusive results with respect to alterations in brain morphology. They assume that chronic cannabis abuse may induce structural changes, whereas in first-episode and high-risk patients, studies need replication. In subjects at high risk and patients with schizophrenia, the gyrification index as a measure of cortico-cortical connection differed from healthy controls in the frontal and parietal cortex [3]. This may be based on the neurodevelopmental abnormalities, since the gyrification pattern is determined during the perinatal period. Accordingly, in a recent postmortem study, a reduced gyrification index has been observed in the parietal cortex in schizophrenia [4] showing no differences between monozygotic twins compared to dizygotic twin pairs which points to the influence of different intrauterine and postnatal factors [5].

Postmortem studies with a resolution on the cellular and even molecular level with, for example, focus on synaptic structural elements [6] may contribute to elucidate the pathophysiology of schizophrenia. Williams et al. [7] investigated neuronal cell density and found decreased pyramidal layer thickness resulting in decreased frontal lobe thickness in schizophrenia patients. They additionally

A. Schmitt $(\bowtie) \cdot$ P. Falkai

Department of Psychiatry and Psychotherapy,

Ludwig-Maximilians-University Munich,

Nußbaumstr. 7, 80336 Munich, Germany

e-mail: Andrea.Schmitt@med.uni-muenchen.de report decreased astrocyte density in the subgenual cingulated cortex and callosal genu for their sample [8], which extends neurobiological hypotheses on the dysfunction of astrocytes in maintaining synaptic stability.

Investigations in healthy subjects are indispensable to elucidate risk factors influencing brain volumes and for exclusion of confounding factors such as other diseaserelated and treatment effects. Beside environmental factors, schizophrenia risk genes may contribute to altered brain structures. Trost et al. [9] found a dysbindin-1 gene variant to be associated with hippocampal and prefrontal gray matter volumes. On the molecular level, another risk gene such as brain-derived neurotrophic factor (BDNF) val66met polymorphism has been shown to affect the glutamate system [10]. Moreover, D-amino acid oxidase activator gene (DAOA) variation has been found to affect homovanillic acid levels in cerebrospinal fluid [11]. Unraveling neurobiological effects of such risk factors may contribute to develop a more personalized medicine in psychiatry [12].

Even effects of traditional neuroleptic treatment strategies may be influenced by genetic factors. Giegling et al. [13] found that three haplotype blocks, one among ankyrin repeat and kinase domain containing 1 (ANKK1) and two among dopamine receptor D2 (DRD2) genes, were associated with better clinical improvement in schizophrenia patients. These often are heavy smokers attempting to treat negative and cognitive symptoms themselves. In a clinical trial investigating effects of transdermal nicotine patch, Chen et al. [14], however, found no correlation with replacement doses and only a low smoking cessation rate. They recommend more effective smoking cessation programs for this group of hospitalized patients.

Due to restricted therapeutic effects of current treatment strategies in severe psychiatric disorders, the editorial 
board of EAPCN decided publishing a series of invited reviews on novel therapeutic approaches in the schizophrenia-affective spectrum, starting in spring 2013. Interested authors are invited to submit proposals (abstracts) to the editor for invited reviews, Prof. Hans-Jürgen Möller.

\section{References}

1. Horga G, Bernacer J, Dusi N et al (2011) Correlations between ventricular enlargement and gray and white matter volumes of cortex, thalamus, striatum, and internal capsule in schizophrenia. Eur Arch Pschiatry Clin Neurosci 261:467-476

2. Malchow B, Hasan A, Fusar-Poli P, Schmitt A, Falkai P, Wobrock $T$ (2013) Cannabis abuse and brain morphology in schizophrenia: a review of the available evidence. Eur Arch Psychiatry Clin Neurosci. doi:10.1007/s00406-012-0346-3

3. Tepest R, Schwarzbach CJ, Krug B, Klosterkötter J, Ruhrmann S, Vogeley K (2013) Morphometry of structural disconnectivity indicators in subjects at risk and in age-matched patients with schizophrenia. Eur Arch Psychiatry Clin Neurosci. doi:10.1007/ s00406-012-0343-6

4. Palaniyappan L, Liddle F (2012) Dissociable morphometric differences in the inferior parietal lobule in schizophrenia. Eur Arch Pschiatry Clin Neurosci 262:579-587

5. Hasan A, McIntosh AM, Droese U-A et al (2011) Prefrontal cortex gyrification index in twins: an MRI study. Eur Arch Pschiatry Clin Neurosci 261:459-465

6. Schmitt A, Leonardi-Essmann F, Durrenberger PF et al (2012) Structural synaptic elements are differentially regulated in superior temporal cortex of schizophrenia patients. Eur Arch Pschiatry Clin Neurosci 262:565-577

7. Williams MR, Chaudhry R, Perera S, Pearce RKB, Hirsch SR, Ansorge O, Thom M, Maier M (2013) Changes in cortical thickness in the frontal lobes in schizophrenia are a result of thinning of pyramidal cell layers. Eur Arch Psychiatry Clin Neurosci. doi:10.1007/s00406-012-0325-8

8. Williams MR, Hampton T, Pearce RKB, Hirsch SR, Ansorge O, Thom M, Maier M (2013) Astrocyte decrease in the subgenual cingulate and callosal genu in schizophrenia. Eur Arch Psychiatry Clin Neurosci. doi:10.1007/s00406-012-0328-5

9. Trost S, Platz B, Usher J, Scherk H, Wobrock T, Ekawardhani S, Meyer J, Reith W, Falkai P, Gruber O (2013) The DTNBP1 (dysbindin-1) gene variant rs2619522 is associated with variation of hippocampal and prefrontal grey matter volumes in humans. Eur Arch Psychiatry Clin Neurosci. doi:10.1007/s00406012-0320-0

10. Gruber O, Hasan A, Scherk H et al (2012) Association of the brain-derived neurotrophic factor val66met polymorphism with magnetic resonance spectroscopic markers in the human hippocampus: in vivo evidence for effects on the glutamate system. Eur Arch Pschiatry Clin Neurosci 262:23-31

11. Andreou D, Saetre P, Werge T et al (2012) D-amino acid oxidase activator gene (DAOA) variation affects cerebrospinal fluid homovanillic acid concentrations in healthy Caucasians. Eur Arch Pschiatry Clin Neurosci 262:549-556

12. Falkai P (2011) Personalized psychiatry and psychotherapy. Realistic hope for the future or fiction? Nervenarzt 82(11): 382-1384

13. Giegling I, Balzarro B, Porcelli S, Schäfer M, Hartmann AM, Friedl M, Konte B, Krämer P, Möller H-J, De Ronchi D, Stassen $\mathrm{HH}$, Serretti A, Rujescu D (2013) Influence of ANKK1 and DRD2 polymorphisms in response to haloperidol. Eur Arch Psychiatry Clin Neurosci. doi:10.1007/s00406-012-0348-1

14. Chen H-K, Lan T-H, Wu BJ (2013) A double-blind randomized clinical trial of different doses of transdermal nicotine patch for smoking reduction and cessation in long-term hospitalized schizophrenic patients. Eur Arch Psychiatry Clin Neurosci. doi: 10.1007/s00406-012-0338-3 